\title{
Hierarchical Modeling of Hydro-Mechanical Coupling in Fractured Shale Gas Reservoirs with Multiple Porosity Scales
}

Xia Yan ${ }^{1}$, Hai Sun ${ }^{1 *}$, Zhaoqin Huang ${ }^{1}$, Lijun Liu ${ }^{1}$, Ping Wang ${ }^{2}$, Qi Zhang ${ }^{3}$, Jun Yao ${ }^{*}$

1 School of Petroleum Engineering, China University of Petroleum (East China), Qingdao 266580, China

2 Shaanxi Key Laboratory of Lacustrine Shale Gas Accumulation and Exploitation (under planning)

3 Department of Civil and Environmental Engineering, Stanford University, Stanford, CA 94305, USA

\section{Appendix A: Homogenization Upscaling at Microscopic Scale}

In this appendix, the homogenization procedure of equivalent mesoscopic model is derived. We now substitute Eq.11 into Eqs.1-3, apply Eq.12, and collect terms with like powers of $\varepsilon$.

The $\varepsilon^{-1}$ terms of Eq. 3 and $\varepsilon^{-2}$ terms of Eq. 1 yield

$$
\nabla_{y} p^{(0)}=0, \quad \nabla_{y} \cdot\left(\boldsymbol{C} \boldsymbol{e}_{y}\left(\boldsymbol{u}_{\mathrm{s}}^{(0)}\right)\right)=0
$$

It can be deduced that

$$
p^{(0)}=p^{(0)}(x, t), \quad \boldsymbol{u}_{\mathrm{s}}^{(0)}=\boldsymbol{u}_{\mathrm{s}}^{(0)}(x, t)
$$

The $\varepsilon^{-1}$ terms of Eq. 2 and $\varepsilon^{0}$ terms of Eq. 3 yield

$$
\left\{\begin{array}{l}
\nabla_{y} \cdot\left(\rho_{g}\left(p^{(0)}\right) \boldsymbol{v}^{(0)}\right)=0 \\
\frac{\mu\left(p^{(0)}\right)}{k_{a}\left(p^{(0)}\right)} \boldsymbol{v}^{(0)}+\nabla_{y} p^{(1)}=-\nabla_{x} p^{(0)}
\end{array}\right.
$$

Therefore, $\boldsymbol{v}^{(0)}$ and $p^{(1)}$ can be written in the form

$$
\left\{\begin{array}{l}
\boldsymbol{v}^{(0)}=-\frac{1}{\mu\left(p^{(0)}\right)} \omega(y) \nabla_{x} p^{(0)} \\
p^{(1)}=-\pi(y) \nabla_{x} p^{(0)}
\end{array}\right.
$$

For each $i$-th dimension $(i=1, \ldots, \mathrm{d})$. With $\boldsymbol{e}_{i}$ being the standard Cartesian basis vector in the $i$-th direction. The $\Omega_{\mathrm{p}}$-periodic vectors $\omega_{i}$ and $\pi_{i}$, of zero volume average over $\Omega_{\mathrm{p}}$, are the solutions of following equations with periodic boundary

$$
\left\{\begin{array}{l}
\nabla_{y} \cdot \omega_{i}=0 \\
\frac{1}{k_{a}\left(p^{(0)}\right)} \omega_{i}+\nabla_{y} \pi_{i}=\boldsymbol{e}_{i}
\end{array}\right.
$$

We now apply the averaging operator in the following sense

$$
\left\langle\boldsymbol{v}^{(0)}\right\rangle_{\Omega_{\mathrm{p}}}=-\frac{1}{\mu\left(p^{(0)}\right)}\left(\frac{1}{\left|\Omega_{\mathrm{p}}\right|} \int_{\Omega_{\mathrm{p}}} \omega\left(p^{(0)}\right) \mathrm{d} \Omega\right) \nabla_{x} p^{(0)}
$$


where $\left|\Omega_{\mathrm{p}}\right|$ denotes the volume of $\Omega_{\mathrm{p}}$ and the symbol $\langle a\rangle$ stands for the volume average of $a$

$$
\langle a\rangle=\frac{1}{|\Omega|} \int_{\Omega} a \mathrm{~d} \Omega
$$

Now let the equivalent mesoscopic permeability $\boldsymbol{k}_{a}^{\mathrm{me}}$ defined as

$$
k_{a i j}^{\mathrm{me}}\left(p^{(0)}\right)=\frac{1}{\left|\Omega_{\mathrm{p}}\right|} \int_{\Omega_{\mathrm{p}}}\left(\omega_{i}\left(p^{(0)}\right)\right){ }_{j} \mathrm{~d} \Omega
$$

Then we can obtain the following mesoscopic Darcy's equation

$$
\left\langle\boldsymbol{v}^{(0)}\right\rangle_{\Omega_{p}}=-\frac{\boldsymbol{k}_{a}^{\mathrm{me}}\left(p^{(0)}\right)}{\mu\left(p^{(0)}\right)} \nabla_{x} p^{(0)}
$$

Eq. 1 at the $\varepsilon^{-1}$ order

$$
\nabla_{y} \cdot\left(\boldsymbol{C}\left(\boldsymbol{e}_{x}\left(\boldsymbol{u}_{\mathrm{s}}^{(0)}\right)+\boldsymbol{e}_{y}\left(\boldsymbol{u}_{\mathrm{s}}^{(1)}\right)\right)\right)=0
$$

Therefore, $\boldsymbol{u}_{\mathrm{s}}^{(1)}$ can be written in the form

$$
\boldsymbol{u}_{\mathrm{s}}^{(1)}=\boldsymbol{\xi}(y) \boldsymbol{e}_{x}\left(\boldsymbol{u}_{\mathrm{s}}^{(0)}\right)+\overline{\boldsymbol{u}}_{\mathrm{s}}^{(1)}(x, t)
$$

where $\overline{\boldsymbol{u}}_{\mathrm{s}}^{(1)}(x, t)$ is an $y$-independent arbitrary displacement. The $\Omega_{\mathrm{p}}$-periodic vectors $\xi^{p q}$, of zero volume average over $\Omega_{\mathrm{p}}$, are the solution of Eq. A.12 with periodic boundary.

$$
\nabla_{y} \cdot\left(C_{i j k l}\left(\tilde{\boldsymbol{e}}_{k l}+\boldsymbol{e}_{y k l}\left(\xi^{p q}\right)\right)\right)=0, \quad \tilde{\boldsymbol{e}}_{k l}=\frac{1}{2}\left(\delta_{k p} \delta_{l q}+\delta_{k q} \delta_{l p}\right)
$$

Consider Eq. 1 at $\varepsilon^{0}$ order

$$
\nabla_{x} \cdot\left(\boldsymbol{C}\left(\boldsymbol{e}_{x}\left(\boldsymbol{u}_{\mathrm{s}}^{(0)}\right)+\boldsymbol{e}_{y}\left(\boldsymbol{u}_{\mathrm{s}}^{(1)}\right)\right)-\alpha p^{(0)} \mathbf{I}\right)+\nabla_{y} \cdot\left(\boldsymbol{C}\left(\boldsymbol{e}_{x}\left(\boldsymbol{u}_{\mathrm{s}}^{(1)}\right)+\boldsymbol{e}_{y}\left(\boldsymbol{u}_{\mathrm{s}}^{(2)}\right)\right)-\alpha p^{(1)} \mathbf{I}\right)=0
$$

Let us integrate Eq. A.12 on $\Omega_{\mathrm{p}}$, and apply the divergence theorem and periodic boundary,

$$
\left\langle\nabla_{x} \cdot\left(\boldsymbol{C}\left(\boldsymbol{e}_{x}\left(\boldsymbol{u}_{\mathrm{s}}^{(0)}\right)+\boldsymbol{e}_{y}\left(\boldsymbol{u}_{\mathrm{s}}^{(1)}\right)\right)-\alpha p^{(0)} \mathbf{I}\right)\right\rangle_{\Omega_{p}}=0
$$

Then we can obtain the mesoscopic effective stress in the form

$$
\nabla_{x} \cdot \sigma^{(0)}=0, \quad \sigma^{(0)}=\boldsymbol{C}^{\mathrm{me}} \boldsymbol{e}_{x}\left(\boldsymbol{u}_{\mathrm{s}}^{(0)}\right)-\alpha^{\mathrm{me}} p^{(0)} \mathbf{I}
$$

where the equivalent tensors $\boldsymbol{C}^{\mathrm{me}}$ and $\alpha^{\mathrm{me}}$ are defined as

$$
C_{i j k l}^{\mathrm{me}}=\left\langle C_{\mathrm{mijkl}}+C_{\mathrm{m} i j m n} e_{y m n}\left(\xi^{k l}\right)\right\rangle_{\Omega_{\mathrm{p}}}, \quad \alpha^{\mathrm{me}}=\langle\alpha\rangle_{\Omega_{p}}
$$

Eq. 2 at the order $\varepsilon^{0}$ gives 


$$
\begin{aligned}
\beta\left(p^{(0)}\right) \frac{\partial p^{(0)}}{\partial t}+\delta(1 & -\phi) \frac{\partial m_{\mathrm{g}}^{(0)}}{\partial t}+\alpha \rho_{\mathrm{g}}\left(p^{(0)}\right) \frac{\partial\left(e_{x i i}\left(\boldsymbol{u}_{\mathrm{s}}^{(0)}\right)+e_{y i i}\left(\boldsymbol{u}_{\mathrm{s}}^{(1)}\right)\right)}{\partial t} \\
& +\left(\nabla_{x} \cdot\left(\rho_{\mathrm{g}}\left(p^{(0)}\right) \boldsymbol{v}^{(0)}\right)+\nabla_{y} \cdot\left(\rho_{\mathrm{g}}\left(p^{(0)}\right) \boldsymbol{v}^{(1)}\right)\right)=0
\end{aligned}
$$

Let us average above equation on $\Omega_{\mathrm{p}}$, and apply the divergence theorem and periodic boundary, then we can get the mesoscopic mass balance in the form

$$
\beta^{\mathrm{me}}\left(p^{(0)}\right) \frac{\partial p^{(0)}}{\partial t}+\frac{\partial m_{\mathrm{g}}^{\mathrm{me}}\left(p^{(0)}\right)}{\partial t}+\alpha^{\mathrm{me}} \rho_{\mathrm{g}}\left(p^{(0)}\right) \frac{\partial \nabla_{x} \cdot\left(\boldsymbol{u}_{\mathrm{s}}^{(0)}\right)}{\partial t}+\nabla_{x} \cdot\left(\rho_{\mathrm{g}}\left(p^{(0)}\right)\left\langle\boldsymbol{v}^{(0)}\right\rangle_{\Omega_{\mathrm{p}}}\right)=0
$$

where the equivalent coefficient $\beta^{\text {me }}$ and $m_{\mathrm{g}}^{\text {me }}$ are defined as

$$
\begin{aligned}
& \beta^{\mathrm{me}}\left(p^{(0)}\right)=\left\langle\frac{\alpha-\phi}{K_{\mathrm{s}}} \rho_{\mathrm{g}}\left(p^{(0)}\right)+\phi \frac{M_{\mathrm{g}}}{R T}\left(\frac{1}{Z\left(p^{(0)}\right)}-\frac{p^{(0)}}{Z\left(p^{(0)}\right)^{2}} \frac{\partial Z\left(p^{(0)}\right)}{\partial p^{(0)}}\right)\right\rangle_{\Omega_{\mathrm{p}}} \\
& m_{\mathrm{g}}^{\mathrm{me}}\left(p^{(0)}\right)=\left\langle\delta(1-\phi) \rho_{\mathrm{r}} \rho_{\mathrm{gstd}} V_{\mathrm{L}} \frac{p^{(0)} / Z\left(p^{(0)}\right)}{p_{\mathrm{L}}+p^{(0)} / Z\left(p^{(0)}\right)}\right\rangle_{\Omega_{\mathrm{p}}}
\end{aligned}
$$

3 